\title{
GMRT observations of the XMM large scale structure survey field ${ }^{\star}$
}

\author{
C. Tasse ${ }^{1}$, H. J. A. Röttgering ${ }^{1}$, P. N. Best ${ }^{2}$, A. S. Cohen ${ }^{3}$, M. Pierre ${ }^{4}$, and R. Wilman ${ }^{5}$ \\ 1 Leiden Observatory, University of Leiden, PO Box 9513, 2300 RA Leiden, The Netherlands \\ e-mail: tasse@strw.leidenuniv.nl \\ 2 SUPA, Institute for Astronomy, Royal Observatory Edinburgh, Blackford Hill, Edinburgh EH9 3HJ, UK \\ 3 US Naval Research Laboratory, Remote Sensing Division, 4555 Overlook Avenue, SW, Code 7213, Washington, DC 20375, USA \\ ${ }^{4}$ CEA/DSM/DAPNIA, Service d'Astrophysique, Saclay, 91191 Gif-sur-Yvette, France \\ 5 Department of Physics, Durham University, South Road, Durham, DH1 3LE, UK
}

Received 20 December 2006 / Accepted 11 May 2007

\section{ABSTRACT}

\begin{abstract}
The low-frequency radio survey of the XMM-Large Scale Structure (XMM-LSS) field aims to study the connection between the extragalactic radio source populations and their environment as traced by X-ray and optical emission. In this paper we present new radio observations of the XMM-LSS field carried out using the Giant Meterwave Radio Telescope at 240 and 610 MHz. These observations complement the observations presented by Cohen at al. (2003, ApJ, 591, 640) and Tasse et al. (2006, A\&A, 456, 791) at 74 and $325 \mathrm{MHz}$ with the Very Large Array. At 240 and $610 \mathrm{MHz}$, we reach noise levels of $\sim 2.5$ and $\sim 0.3 \mathrm{mJy} / \mathrm{beam}$, leading to the detection of 466 and 769 sources over 18.0 and 12.7 degree $^{2}$ with resolutions of $14.7^{\prime \prime}$ and $6.5^{\prime \prime}$ respectively. Combining these data with the available source lists at 74, 325 (Tasse et al. 2006, A\&A, 456, 791) and $1400 \mathrm{MHz}$ (NVSS), we build a multifrequency catalogue containing 1611 radio sources. We check for consistency of the astrometry and flux density estimates. We fit a simple synchrotron radiation model to the flux density measurements of the 318 radio sources being detected in at least 4 bands. While $\sim 26 \%$ of them show signature of spectral ageing, $\sim 6 \%$ show self absorption.
\end{abstract}

Key words. techniques: interferometric - methods: statistical - surveys - galaxies: active - radio continuum: galaxies cosmology: large-scale structure of Universe

\section{Introduction}

The XMM-Large Scale Structure survey (XMM-LSS) is a 10 degree $^{2} \mathrm{X}$-ray survey designed to map out the large scale structure of the Universe through the detection of the X-ray emission of the diffuse intracluster medium (ICM). Spectroscopic follow-up of $\sim 70$ galaxy cluster candidates found in the first $\sim 5$ degree $^{2}$ is completed, leading to a galaxy cluster density of $\sim 12 \mathrm{deg}^{-2}$ in the redshift range $z \lesssim 1$ (Pierre et al. 2006; Pacaud et al. 2006), in preparation). Also some $1000 \mathrm{deg}^{-2}$ $\mathrm{X}$-ray active galactic nuclei have been detected.

The Canada France Hawaii Telescope Legacy Survey $\left(\right.$ CFHTLS $\left.^{1}\right)$ has observed the XMM-LSS field in 5 bands down to $i_{\mathrm{AB}} \sim 25$, allowing for optical identification of X-ray sources and determination of photometric redshifts. Also, the XMM-LSS field was imaged in 7 bands from 3 to $160 \mu \mathrm{m}$ as part of the Spitzer Wide-area Infrared Extragalactic Survey (SWIRE, Lonsdale et al. 2003). For a general overview of the XMM-LSS and associated surveys see Pierre et al. (2004).

Cohen et al. (2003) and Tasse et al. (2006) have conducted a deep low-frequency survey of the XMM-LSS field at 74 and $325 \mathrm{MHz}$ with the Very Large Array (VLA), reaching flux density limits of $\sim 162$ and $\sim 4 \mathrm{mJy} /$ beam $(5 \sigma)$, leading to the detection of $\sim 1500$ radio sources over $\sim 130$ and $\sim 15$ degree $^{2}$ with resolutions of $30^{\prime \prime}$ and $6.7^{\prime \prime}$ respectively. The main scientific

\footnotetext{
* Tables B.1 and B.2 are only available in electronic form at the CDS via anonymous ftp to cdsarc.u-strasbg.fr $(130.79 .128 .5)$ or via

http://cdsweb.u-strasbg.fr/cgi-bin/qcat?J/A+A/471/1105

1 for more information on the CFHT Legacy Survey, see http://www.cfht .hawaii.edu/Science/CFHLS/
}

issues that such data can address include: (1) Where are various radio-loud active galactic nuclei (AGN) located with respect to the distribution of mass on cosmological scales as traced by the optical and X-Ray emission? (2) Can the radio loudness of optical and X-ray AGN be understood as due to environmental effects? (3) How does the hot IGM influence the physical properties of the radio sources such as linear size and radio power?

In order to increase the size of the low frequency radio sources sample (Tasse et al. 2006), we have conduced new observations of the XMM-LSS field at 240 and $610 \mathrm{MHz}$ using the Giant Meterwave Radio Telescope (GMRT). As compared to the VLA, the GMRT has a collecting area a factor of 3 bigger. Integration time of $\sim 20 \mathrm{~h}$ at each frequency, lead to the detection of $\sim 200$ additional radio sources over $\sim 15$ degree $^{2}$, corresponding to an increase of the sample size of $\sim 25 \%$.

In addition, for $41 \%$ of the radio sources detected at $325 \mathrm{MHz}$, our GMRT observations provide two additional flux density points giving the opportunity to study the links between host galaxy, environment, and observed radio spectra for large samples of radio sources. Specifically, within an evolutionary framework, the compact steep spectrum (CSS) and gigahertz peaked spectrum (GPS) radio sources showing signs of synchrotron self absorption at $v<1 \mathrm{GHz}$, are thought to be the progenitors of larger steep spectrum FRI and/or FRII (Fanaroff \& Riley 1974) radio sources (e.g. Snellen et al. 2003; O'Dea 1998). When no more high energy electrons are injected into the synchrotron emitting lobes, spectral ageing feature appears in the radio spectra. If the link between these different classes of radio sources is effectively evolutionary, they should be found in similar environments, while the infrared and optical properties of the host galaxy should be evolving. The large multiwavelength 

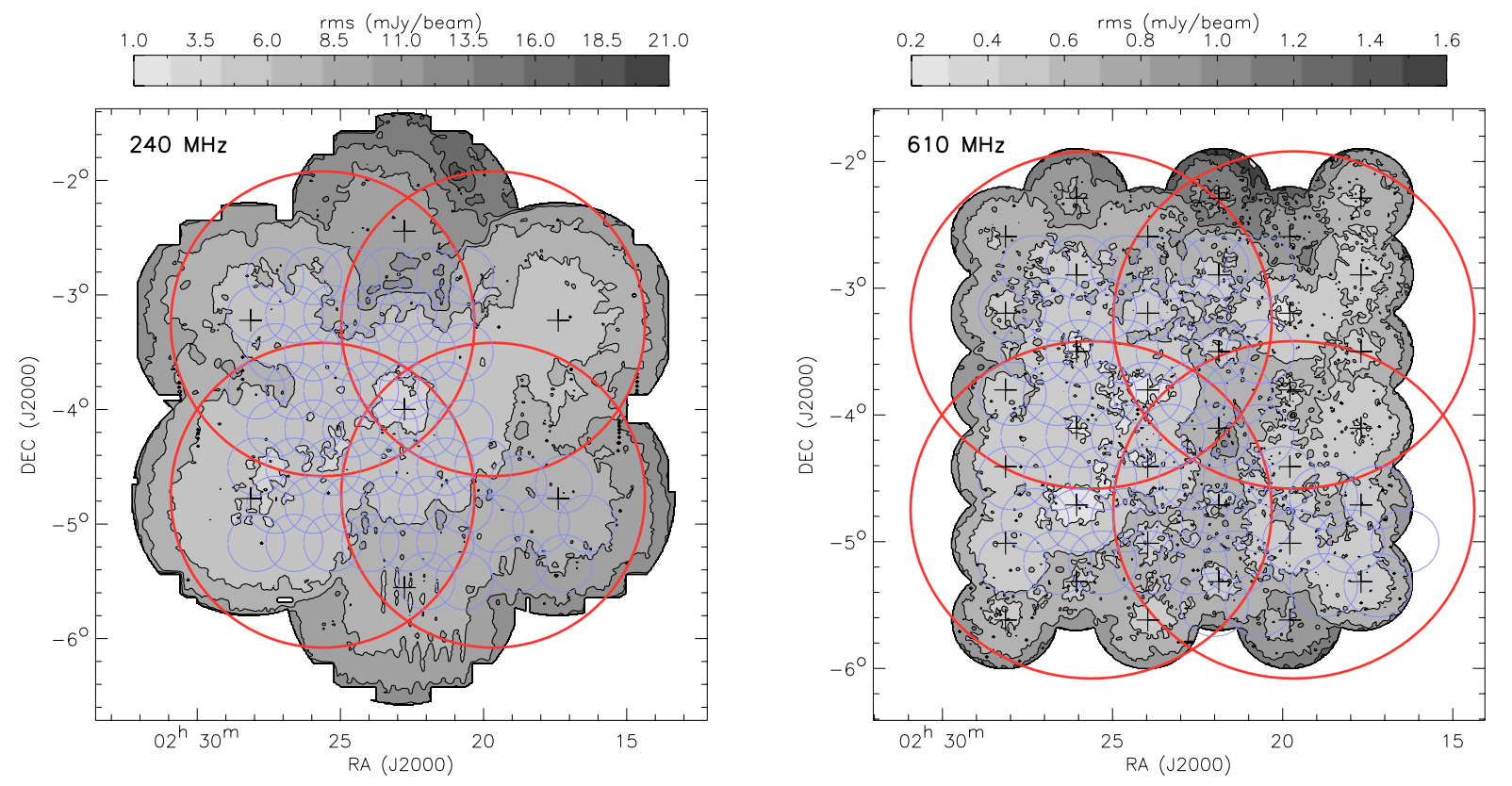

Fig. 1. The noise maps at 240 and $610 \mathrm{MHz}$. On both panels, contours where plotted at level of $\sigma \times\{1,1.4,2,2.8,4,5.6,8 \ldots\}$ with $\sigma=1.0 \mathrm{mJy}$ and $\sigma=0.2 \mathrm{mJy}$ at 240 and $610 \mathrm{MHz}$ respectively. On both panels, the crosses show the location of each individual pointing center, the thick red line shows the four $325 \mathrm{MHz}$ VLA pointings, and the blue circles show the location of the X-ray pointing. The primary beams at $240 \mathrm{MHz}$ are counter intuitively smaller than those of the VLA at $325 \mathrm{MHz}$ because the GMRT antennas are larger.

Table 1. Observational parameters for the XMM-LSS radio followup with GMRT.

\begin{tabular}{ccc}
\hline \hline Central frequency & $40 \mathrm{MHz}$ & $610 \mathrm{MHz}$ \\
\hline Obs. date & $30,31 \mathrm{Jul} .$, & $30,31 \mathrm{Jul}$. \\
& $3 \mathrm{Aug} .2004$ & $1,2 \mathrm{Aug} .2004$ \\
\hline Number of pointings & 7 & 36 \\
Int. time per pointing & $\sim 2.8 \mathrm{~h}$ & $\sim 0.5 \mathrm{~h}$ \\
Total obs. time $(\mathrm{h})$ & $\sim 20$ & $\sim 18$ \\
\hline Band Width & $8 \mathrm{MHz}$ & $2 \times 16 \mathrm{MHz}$ \\
$N_{\text {channel }}$ & 128 & $2 \times 128$ \\
UV range $(\mathrm{k} \lambda)$ & $\sim 0.3-18$ & $\sim 0.3-48$ \\
\hline Band Pass Cal. & $3 \mathrm{C} 48$ & $3 \mathrm{C} 48$ \\
Phase Cal. & $0116-208$ & $0116-208$ \\
\hline
\end{tabular}

dataset available on the XMM-LSS field, combined with the various radio surveys we have undertaken may allow us to address this issue in a consistent way.

In this paper we present the GMRT radio observations, and discuss their consistency. Using our multifrequency dataset and a simple model of synchrotron emission we build subsamples of sources showing self absorption and/or spectral ageing features. In subsequent papers, we will address the main scientific issues using the infrared, optical and X-ray data available on the XMM-LSS field.

This paper is organised as follow: in Sect. 2 we give details of the observations, and data reduction. In Sect. 3 we briefly describe the source extraction methods, and we present the error analysis in Sect. 4. The final source list is presented and analysed in Sect. 5. In Sect. 6 we build a multifrequency radio sample and we fit a simple synchrotron radiation model to the brightest sources to build the self absorbed and spectrally aged subsamples. We conclude in Sect. 7.

\section{Observations and data reduction}

\subsection{Radio continuum at $610 \mathrm{MHz}$}

The XMM-LSS field centered at $\alpha(\mathrm{J} 2000)=2^{\mathrm{h}} 24^{\mathrm{m}} 00^{\mathrm{s}}$, $\delta(\mathrm{J} 2000)=-4^{\circ} 09^{\prime} 47^{\prime \prime}$ was observed at $610 \mathrm{MHz}$ from the 30th of July to the 2nd of August 2004 for a total of $\sim 18 \mathrm{~h}$. We have used the full available bandwidth of $32 \mathrm{MHz}$, split into two intermediate frequencies (IFs), each IF being sampled into 128 channels. The first and second IF were covering the intervals 594-610 MHz and 610-626 MHz, respectively (see Table 1 for an overview). At $610 \mathrm{MHz}$ the GMRT full width at half maximum ( $F W H M$ i.e. primary beam diameter) is $\theta_{F W H M} \sim 0.7^{\circ}$. In order to survey the $\sim 15$ degree $^{2}$ of the $325 \mathrm{MHz}$ counterpart of the XMM-LSS field (Tasse et al. 2006), we spread the pointing centers over an hexagonal grid consisting of 36 pointings centers separated by $0.6^{\circ}$ (Fig. 1 ). The pointing grid covers $78 \%$ of the area mapped at $325 \mathrm{MHz}$. For each pointing, we have used a total integration time of $30 \mathrm{~min}$. Since the GMRT array consists of three arms, in the $u v$-plane the visibilities corresponding to each individual baseline come back to the same $u v$-location every $4 \mathrm{~h}$. In order to optimize the $u v$-plane coverage we split each $30 \mathrm{~min}$ observation of each individual pointing into three $10 \mathrm{~min}$ scans, separated by about $1.3 \mathrm{~h}$. For the flux and bandpass calibration we used 3C 48 as flux density and bandpass calibrator, observed for $30 \mathrm{~min}$ at the beginning and the end of the run. We used 0116-208 as phase and amplitude calibrator, observed for $8 \mathrm{~min}$ every $30 \mathrm{~min}$.

For the data reduction work, we made use of the Astronomical Image Processing System (AIPS). We reduced each IF independently. After a preliminary round of Radio Frequency Interference (RFI) removal using AIPS tasks SPFLG and UVFLG, the bandpass as well as the overall flux density scale were calibrated using $3 \mathrm{C} 48$, and the amplitudes of the gains of each antenna were calibrated using 0116-208. The expected resolution of $\sim 6.5^{\prime \prime}$ is similar to the $6.7^{\prime \prime}$ resolution obtained at $325 \mathrm{MHz}$ with VLA. In order to calibrate 


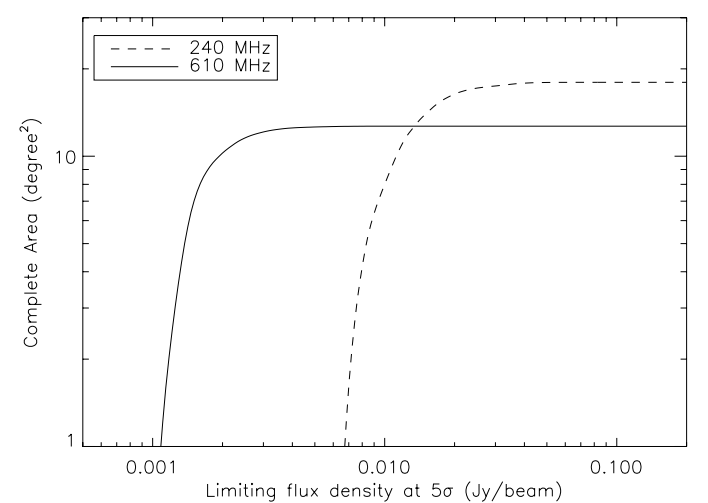

Fig. 2. Area as a function of limiting flux density at $5 \sigma$. The full line corresponds to the $610 \mathrm{MHz}$ survey whereas the dashed one corresponds to the $240 \mathrm{MHz}$ survey.

the phases variation with time, we build from the Tasse et al. (2006) $325 \mathrm{MHz}$ source list a catalog containing the positions and flux densities of all individual components brighter than $10 \mathrm{mJy}$. Most of these $325 \mathrm{MHz}$ sources have positional uncertainties on the level of 1.5". By Fourier transforming this model of the sky plane, the AIPS task CALIB generates a corresponding model of the $u v$-plane that is used to calibrate the phase of each antenna every $3 \mathrm{~min}$. This approach provides the advantage of suppressing all possible astrometry offsets between the different pointings.

In order to image the full primary beam, we have used a pseudo three dimensional Fourier transform technique (Perley 1999), where each pointing is divided into smaller fields (facets). We have used $\sim 20$ facets per pointing each $13^{\prime}$ in size. Each facet was then imaged, and combined into a single image using AIPS task FLATN. The fitted size of the dirty beam is $\sim 6.5^{\prime \prime}$ along the major axis and $\sim 4.5^{\prime \prime}$ along the minor axis. In order to have a homogeneous beam across the different pointings in the combined map, the restoring beam was set to be a $6.5^{\prime \prime} \times 6.5^{\prime \prime}$ circular Gaussian in each pointing, angular scale being $1.5^{\prime \prime} \mathrm{pixel}^{-1}$. After few standard phase only self-calibration steps, the synthesized images of the two IFs of each pointing have been averaged. Using a radial cutoff limit of $0.35^{\circ}$ (corresponding to the $F W H M$ ), the 2 IFs $\times 36$ pointings have been combined into a $\sim 13$ degree $^{2}$ single image following the procedure described by Condon et al. (1998). In the final map the noise is as low as $\sim 0.2 \mathrm{mJy}$ beam $^{-1}$ to as high as $\sim 1.5 \mathrm{mJy}^{\text {beam }}{ }^{-1}$ (Fig. 1 ).

\subsection{Radio continuum at $240 \mathrm{MHz}$}

The data at $240 \mathrm{MHz}$ were obtained using the GMRT from the 30 th of July to the 3rd of August 2004 for a total time of $\sim 20 \mathrm{~h}$. As opposite to the $610 \mathrm{MHz}$ setting, at $240 \mathrm{MHz}$, the $8 \mathrm{MHz}$ wide bandwidth (128 channels) is centered at $240 \mathrm{MHz}$ and consists of only one IF (see Table 1 for an overview). The primary beam diameter being $1.8^{\circ}$, in order to cover the $325 \mathrm{MHz}$ counterpart of the XMM-LSS field, we made use of an hexagonal grid, consisting of 7 pointing centers, separated by $1.56^{\circ}$, as shown in Fig. 1. The pointing grid covers $\sim 93 \%$ of the area mapped at $325 \mathrm{MHz}$. As for the $610 \mathrm{MHz}$ observations, we used $3 \mathrm{C} 48$ at the beginning and the end of each run as flux density and bandpass calibrator, and 0116-208 as phase and amplitude calibrator. Also in order to optimize the $u v$-plane coverage, each pointing was observed for 10 min every $\sim 1.4 \mathrm{~h}$, while the secondary calibrator was observed every $0.5 \mathrm{~h}$ for $8 \mathrm{~min}$. Each

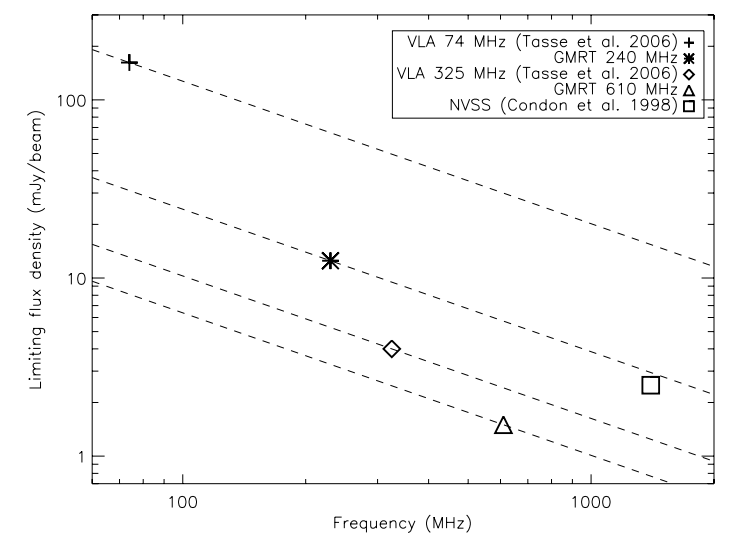

Fig. 3. Flux density limits $(5 \sigma)$ reached at 240 and $610 \mathrm{MHz}$ as compared to the flux density reached at 74 and $325 \mathrm{MHz}$ with VLA. Dashed lines show the flux density of a source having a spectral index of $\alpha=-0.8$.

pointing has been observed $\sim 2.8 \mathrm{~h}$ in total. Table 1 shows a summary of the observational parameters we have been using.

We have synthesized the $240 \mathrm{MHz}$ images as we did at $610 \mathrm{MHz}$. As for the $610 \mathrm{MHz}$ data, we calibrated the phases using a model derived from the source list at $325 \mathrm{MHz}$ by Tasse et al. (2006) (see Sect. 2.1). The restoring beam has been set to be 14.7" circular Gaussian in each map, with a pixel size of $3.5^{\prime \prime}$ pixel $^{-1}$. After a few phase-only self calibration steps we have combined the 7 pointings into a single map using a radial cutoff of $0.9^{\circ}$. The resulting noise varies from as low as $1.2 \mathrm{mJy} / \mathrm{beam}$ and as high as $10 \mathrm{mJy} /$ beam close to bright sources.

At both 240 and $610 \mathrm{MHz}$, Fig. 2 shows the complete area as a function of the sensitivity $(5 \sigma)$, and Fig. 3 shows the limiting flux density in the different available frequency bands.

\section{Source extraction}

For a detailed discussion on the method we have used to extract the sources from the synthesized maps, see Tasse et al. (2006). We briefly describe that process below.

With the local noise being highly variable across the field (see Figs. 1 and 2), special attention was paid to the source finding method. First, a local noise map is generated using AIPS task RMSD, which derives a local noise value within a box. Given the noise distribution is non-Gaussian, the size of the box is critical. We set visually the size of this box to the scale over which the local noise varies significantly, which leads to values of $3^{\prime}$ and $1^{\prime}$ at 240 and $610 \mathrm{MHz}$ respectively.

Then we normalize the combined map by the local noise map, and we extract the sources in that noise-normalized map using AIPS's Gaussian fitting algorithm Search And Destroy (SAD), giving an input cut on both the peak and the integrated flux densities of 5 (corresponding to $5 \sigma_{\text {local }}$ in the original map). We then correct for the peak flux bias introduced by the noise normalization, and calculate the errors on each Gaussian fitting parameter following Condon (1997).

Following Tasse et al. (2006), we build a catalog by associating the components of the source list closer than $60^{\prime \prime}$. The number of Gaussian fitting components being 571 and 1024 at 240 and $610 \mathrm{MHz}$ respectively, assuming a Poisson statistics, the probability of two components to be associated by chance is less than $1 \%$ in both cases. In the catalogs appearing in Tables B. 1 and B.2, we have tagged the brightest component of multiple 
sources with an " $M$ ", and the other components with a "C". Non associated components have been classified as Single sources (tag "S").

\section{Determination of source parameters and associated errors}

\subsection{Astrometry}

The XMM-LSS field being fully covered at $1.4 \mathrm{GHz}$ by the NVSS (Condon et al. 1998) we have looked for $1.4 \mathrm{GHz}$ NVSS sources in a $45^{\prime \prime}$ radius around each individual radio source $^{2}$. For the sources identified in the NVSS, this provides $1.4 \mathrm{GHz}$ astrometry, as well as a spectral index estimate. To assess the positional accuracy, in our catalog we have selected a subsample of single ("S") sources with their NVSS counterpart being point-like and having positional uncertainties $\sigma_{\alpha, \delta}^{\text {NVSS }}<$ $0.7^{\prime \prime}$ on both right ascension and declination, which has the effect of selecting the radio sources that have the brightest $1.4 \mathrm{GHz}$ counterparts. On this subsample containing 33 sources, we measure overall astrometrical offsets of $\Delta\left(\alpha_{610}\right)=1.15^{\prime \prime}$ and $\Delta\left(\delta_{610}\right)=-1.50^{\prime \prime}$ at $610 \mathrm{MHz}$ on right ascension and declination respectively, and $\Delta\left(\alpha_{240}\right)=2.84^{\prime \prime}$ and $\Delta\left(\delta_{240}\right)=-1.85^{\prime \prime}$ at $240 \mathrm{MHz}$. These offsets are on the order of the pixel size at both 240 and $610 \mathrm{MHz}$. We have corrected for these astrometry shifts in the final catalog. In order to be conservative, given the fitted Gaussian major and minor axis and their associated error bars, we have classified as unresolved the sources that are indistinguishable from the restoring beam size at the $2 \sigma$ level $^{3}$.

From the same high signal-to-noise subsample, we measure the standard deviation of the position differences between fitted components and their NVSS counterpart to be $\sigma_{\alpha}=0.70^{\prime \prime}$ and $\sigma_{\delta}=1.78^{\prime \prime}$ at $610 \mathrm{MHz}$ and $\sigma_{\alpha}=1.31^{\prime \prime}$ and $\sigma_{\delta}=2.37^{\prime \prime}$ at $240 \mathrm{MHz}$. These errors contain a contribution from the positional uncertainties in our catalog and a contribution from the NVSS positional uncertainties. In both these components, there is a contribution from the Gaussian fitting errors, which depends on the signal-to-noise ratio of the detection, and a contribution from the individual antenna calibration errors, which does not depend on the local rms value. In order to derive an estimate of the positional calibration errors in our GMRT data $\sigma_{\text {GMRTcal }}$ on both right ascension, and declination we write:

$\sigma^{2}=\sigma_{\text {NVSScal }}^{2}+\sigma_{\text {NVSSfit }}^{2}+\sigma_{\text {GMRTcal }}^{2}+\sigma_{\text {GMRTfit }}^{2}$.

For the NVSS the positional errors due to the antenna calibration has been measured to be $\sigma_{\alpha, \mathrm{NVSS}} \mathrm{Nal}=0.45^{\prime \prime}$ and $\sigma_{\delta, \mathrm{NVSScal}}=$ $0.56^{\prime \prime}$ (Condon et al. 1998). In the high signal-to-noise ratio subsample defined above, corresponding to the brightest sources in our source list, we measure the associated Gaussian fitting errors on position $\sigma_{\text {GMRT,fit }} \lesssim 0.1^{\prime \prime}$ at both 240 and $610 \mathrm{MHz}$ making the Gaussian fitting error contribution to be negligible. We derive an upper limit to the residual systematic errors in the $610 \mathrm{MHz}$ astrometry of $\sigma_{\alpha, \text { GMRTcal }} \lesssim\left(\sigma_{\alpha}^{2}-\sigma_{\alpha, \text { NVSScal }}^{2}\right)^{1 / 2}=0.53^{\prime \prime}$ and $\sigma_{\delta \text {, GMRTcal }} \lesssim 1.69^{\prime \prime}$. At $240 \mathrm{MHz}$ we find $\sigma_{\alpha \text {,calib }} \lesssim 1.23^{\prime \prime}$ and $\sigma_{\delta \text {,calib }} \lesssim 2.31^{\prime \prime}$. We quadratically add these values to the error obtained from the Gaussian fits.

\footnotetext{
${ }^{2}$ Note that we can not use the deeper and higher resolution $1.4 \mathrm{GHz}$ FIRST survey data (Becker et al. 1995) because it only partly covers the XMM-LSS field.

${ }^{3}$ If $\theta_{\mathrm{N}}$ is the restoring beam diameter, $\theta_{\mathrm{M}}$ the major axis diameter, and $\sigma\left(\theta_{\mathrm{M}}\right)$ its associated error bar, then the fitted component is classified as unresolved if $\left(\theta_{\mathrm{N}}-\theta_{\mathrm{M}}\right)^{2} / \sigma^{2}\left(\theta_{\mathrm{M}}\right)<2$. Same for the minor axis.
}

\subsection{Flux densities}

Various factors have to be taken into account in order to derive source lists having reliable flux densities estimates.

Contrarily to the VLA, at the GMRT the injection of calibrated noise at the front-end of each antenna to measure the system temperature has not been implemented. To calibrate the gains of each individual antenna, the system temperature $T_{\text {sys }}$ is assumed to be sum of various components:

$T_{\text {sys }}=T_{\mathrm{r}}+T_{\mathrm{a}}+T_{\text {sky }}$

$T_{\text {sky }}=3+20(408 / v)^{2.75}$

where $T_{\mathrm{r}}$ is the receiver temperature, $T_{\mathrm{a}}$ is the antenna temperature, $T_{\text {sky }}$ is the sky temperature and $v$ is the observed frequency. At each individual frequency, $T_{\mathrm{r}}$, and $T_{\mathrm{a}}$ are assumed to be independent of the pointed position ${ }^{4}$. However, because of the Galactic diffuse radio emission, the sky temperature can vary greatly from one position to another, with $T_{\text {sky }}$ being higher in the galactic plane. The gain by system temperature in units of $\mathrm{K} \mathrm{Jy}^{-1}$ used to calibrate the flux densities of the data, is based on the measurement $T_{\text {sys }}$ at the position of the flux density calibrator. If $T_{\text {sys }}^{\prime}$ is the system temperature at the position of the field, then an overall multiplicative bias $T_{\text {sys }} / T_{\text {sys }}^{\prime}$ is introduced on the flux density estimates at the location of the field. The flux density calibrator $3 \mathrm{C} 48$ that we have used is situated at $b=133.96^{\circ}$ and $l=-28.71^{\circ}$ (in Galactic coordinates), whereas the field is centered around $l \sim 173^{\circ}$ and $l \sim-57^{\circ}$. Using the Haslam et al. (1982) all-sky radio maps at $408 \mathrm{MHz}$, that have a resolution of $0.85^{\circ}$, we estimate using Eq. (3) that the flux densities need to be corrected by $\sim-2 \%$ at $610 \mathrm{MHz}$ and $\sim-18 \%$ at $240 \mathrm{MHz}$. We have corrected for these overall offsets in the final source list.

In the case of resolved sources, calculating the integrated flux density by summing the individual Gaussian fitted flux density components produces a bias towards lower fluxes as compared to the real integrated flux density. Instead, following Tasse et al. (2006) we estimate the flux densities at both 240 and $610 \mathrm{MHz}$ by summing the pixel values in $60^{\prime \prime}$ diameter apertures centered at the position of the individual Gaussian components. If $S_{\text {pix }}$ is the flux density thereby computed, then $S_{\text {pix }}=\Sigma \times 4 \ln 2 /(\pi . F W H M)$, and $\sigma_{\text {pix }}=N_{\text {pix }} \sigma_{\text {loc }}$, where $\Sigma$ is the sum of the pixels inside the aperture, $N_{\text {pix }}$ is the number of pixel over which the summation is done, $\sigma_{\text {loc }}$ is the local noise at the location of the source and FWHM is the Full Width at Half Maximum of the restoring beam in pixels. Because this flux density estimator does not use any a priori knowledge on the restoring beam shape, the error bars associated to the integrated flux density estimate are much bigger than those associated with the Gaussian fitting method. Given the values and error bars estimates for the two methods, we consider the pixel-based estimate of the integrated flux density only when the difference is significant at the $2 \sigma$ level $^{5}$.

Finally, because we have used the secondary calibrator 0116-208 to calibrate the gains of individual antennas, we quadratically add a standard $10 \%$ overall flux density scale error to the noise-based Gaussian fitting uncertainties. This value takes into account any systematic error in the GMRT measurement, like small elevation dependant errors (for a more detailed see Mohan et al. 2001; Chandra et al. 2004).

\footnotetext{
4 See http://www.gmrt.ncra.tifr.res.in for more information on the estimation of each of these temperature.

5 With $\sigma$ being computed as $\sigma=\left(S_{\text {pix }}-S_{\text {gauss }}\right) / \sqrt{\sigma_{\text {pix }}^{2}+\sigma_{\text {gauss }}^{2}}$.
} 

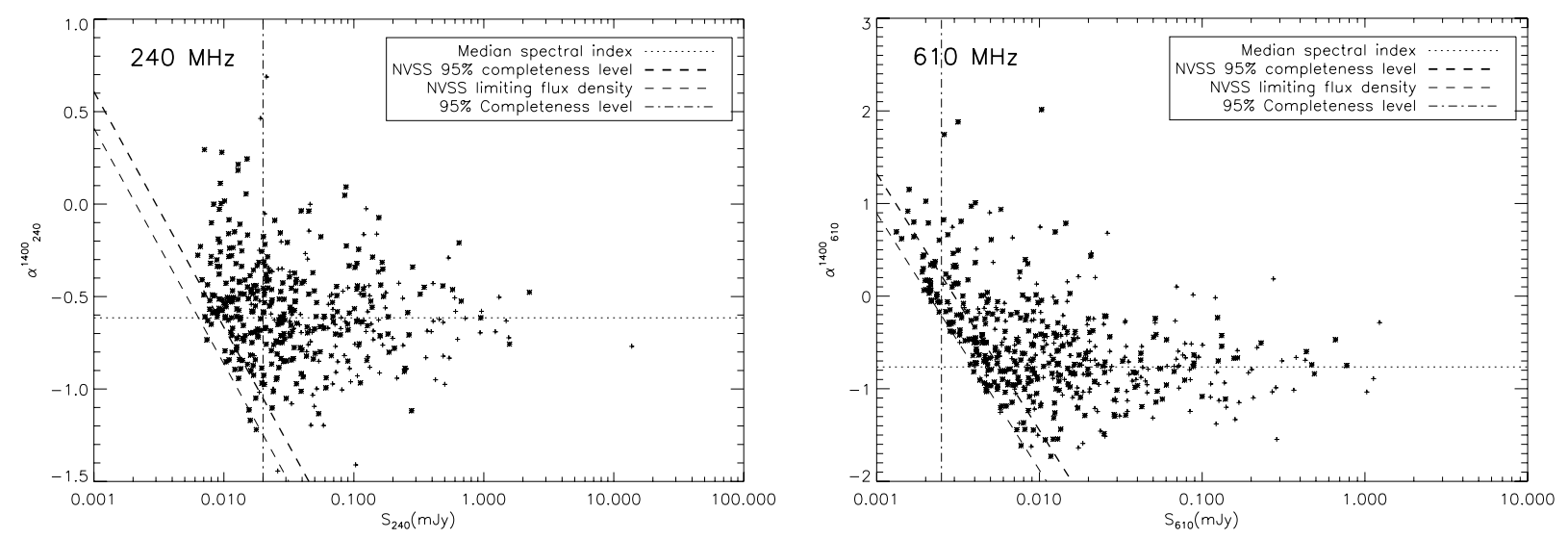

Fig. 4. The spectral index distribution at 240 and $610 \mathrm{MHz}$, derived from flux density comparison with NVSS. The resolution differences at both frequencies (14.7" and 6.5" respectively as compared to 45" for NVSS) leads to an overestimate of the spectral indices for extended and resolved sources, as we may miss a significant fraction of the total flux density. Simple crosses stand for the resolved sources, whereas thick stars are the point-like sources, where no extended emission is expected to bias the spectral index estimate toward higher values. On both panels, the vertical dash-dotted line indicates the $95 \%$ completeness level. On both graphs, horizontal dotted lines indicate the median spectral index value derived from subsamples having integrated flux density $S_{240}^{\text {int }}>63 \mathrm{mJy}$ and $S_{610}^{\text {int }}>30 \mathrm{mJy}$, corresponding to a limiting integrated flux density at $325 \mathrm{MHz}$ of $S_{325}^{\text {int }}>50 \mathrm{mJy}$. Diagonal dashed lines indicate the bias introduced by the limiting flux density and the $95 \%$ completeness limit (thick dashed) of NVSS respectively (Condon et al. 1998). We have given the sources that are not detected in the NVSS, a spectral index upper limit. These points are not plotted, but lie along the $95 \%$ completeness limit of NVSS.

Table 2. Main results in both 240 and $610 \mathrm{MHz}$ bands.

\begin{tabular}{ccc}
\hline \hline Frequency & $240 \mathrm{MHz}$ & $610 \mathrm{MHz}$ \\
\hline Resolution & 14.7 " & 6.5 " \\
Area (degree ${ }^{2}$ ) & 18.0 & 12.7 \\
Rms (mJy/Beam) & $\sim 2.5$ & $\sim 0.3$ \\
\hline Single sources "S" & 388 & 592 \\
Single unresolved & $96 \%$ & $75 \%$ \\
Multiple sources "M" & 79 & 175 \\
\hline
\end{tabular}

\section{Results}

The main characteristics of the 240 and $610 \mathrm{MHz}$ surveys are given in Table 2. The associated source lists are given in Tables B.1 and B.2 respectively. Contour plots are shown in Fig. A. 1 at $240 \mathrm{MHz}$ and in Fig. A. 2 at $610 \mathrm{MHz}$ for the brightest extended sources.

Of the sources detected at $325 \mathrm{MHz}$ (Tasse et al. 2006), $\sim 50 \%$ and $\sim 67 \%$ are detected at 240 and $610 \mathrm{MHz}$ respectively. The fraction of the $325 \mathrm{MHz}$ area surveyed at 240 and $610 \mathrm{MHz}$, is $\sim 93 \%$ and $\sim 78 \%$ respectively. Of the sources detected at $325 \mathrm{MHz}$ and covered by the 240 and $610 \mathrm{MHz}$ surveys, $\sim 55 \%$ and $\sim 80 \%$ are detected at 240 and $610 \mathrm{MHz}$ respectively. Although, the $610 \mathrm{MHz}$ survey is on average deeper by a factor of $\sim 1.5$ than the $325 \mathrm{MHz}$ survey (Fig. 3), the fact that we miss $\sim 20 \%$ of the $325 \mathrm{MHz}$ sources at $610 \mathrm{MHz}$ is explainable because the $325 \mathrm{MHz}$ sources that are missed at $610 \mathrm{MHz}$ are the faintest ones. In order to check for the consistency of the amount of the $325 \mathrm{MHz}$ sources detected at 240 and $610 \mathrm{MHz}$, we first generate a $325 \mathrm{MHz}$ catalog in which each source is given a random flux density value according to their measured flux density and associated error bars. Then each of these source is given a random spectral index following a normal law with mean value $\langle\alpha\rangle=-0.8$ and dispersion $\sigma(\alpha)=0.24$ (De Breuck et al. 2000). We extrapolate the flux density of each of these $325 \mathrm{MHz}$ radio source to 240 and $610 \mathrm{MHz}$, and add up the local noise corresponding to their individual position at 240 and $610 \mathrm{MHz}$. From these simulated 240 and $610 \mathrm{MHz}$ catalog, we extract the respective source lists using a $5 \sigma_{\text {local }}$ cut as we did on the actual maps
(Sect. 3). We observe that such a $325 \mathrm{MHz}$ population would lead to the detection of $\sim 55 \%$ and $\sim 76 \%$ of the of $325 \mathrm{MHz}$ sources at 240 and $610 \mathrm{MHz}$ respectively, in agreement with our observations.

Figure 4 shows the spectral index distribution derived from the flux density comparison with NVSS. The sources without a $1.4 \mathrm{GHz}$ counterpart have been given an upper limit on their spectral index, using the NVSS 95\% completeness limit at $5 \sigma$. At both frequencies the resolutions being 14.7" and 6.5" are smaller than the $45^{\prime \prime}$ resolution of NVSS (Condon et al. 1998). Such differences make the estimation of spectral index to be less reliable for extended radio emission.

In order to compare the spectral index distribution of the 240 and $610 \mathrm{MHz}$ sources to the spectral index distribution of the WENSS $325 \mathrm{MHz}$ radio sources (Rengelink et al. 1997), we assume a mean spectral index of $\alpha_{325}^{1400}=-0.8$ (De Breuck et al. 2000). We extrapolate the limiting integrated flux density of WENSS being $S_{325}^{\lim }=50 \mathrm{mJy}$ to 240 and $610 \mathrm{MHz}$, which makes $S_{240}^{\lim }=63 \mathrm{mJy}$ and $S_{610}^{\lim }=30 \mathrm{mJy}$. We derive median spectral index values of $\alpha_{240}^{1400}=-0.62$ and $\alpha_{610}^{1400}=-0.76$. Figure 5 shows the spectral index distribution of the subsamples defined above $\left(S_{240}=65 \mathrm{mJy}\right.$ and $\left.S_{610}>30 \mathrm{mJy}\right)$ to the WENSS spectral index distribution at $325 \mathrm{MHz}$ (De Breuck et al. 2000). At $240 \mathrm{MHz}$, the spectral index distribution has the same width but is shifted towards shallower spectral indices. This is likely to be due to the combination of the resolution differences (14.7" at $240 \mathrm{MHz}$, against $\sim 55^{\prime \prime}$ for WENSS), and the natural tendency of radio sources to be synchrotron self absorbed at low frequency. At $610 \mathrm{MHz}$, we find close agreement in the distribution centroid, but we note an excess of flat spectrum sources, whose presence might be due to the resolution difference being even bigger, and the shallower effective depth of WENSS to flat spectrum sources.

Below the corresponding flux density limit of WENSS, we detect a few $\alpha>0$ inverted spectral index for unresolved sources at rather small flux densities (Fig. 4). This is expected when synchrotron self absorption occurs in compact radio sources such as compact steep spectrum (CSS) and gigahertz peaked spectrum sources (GPS). 


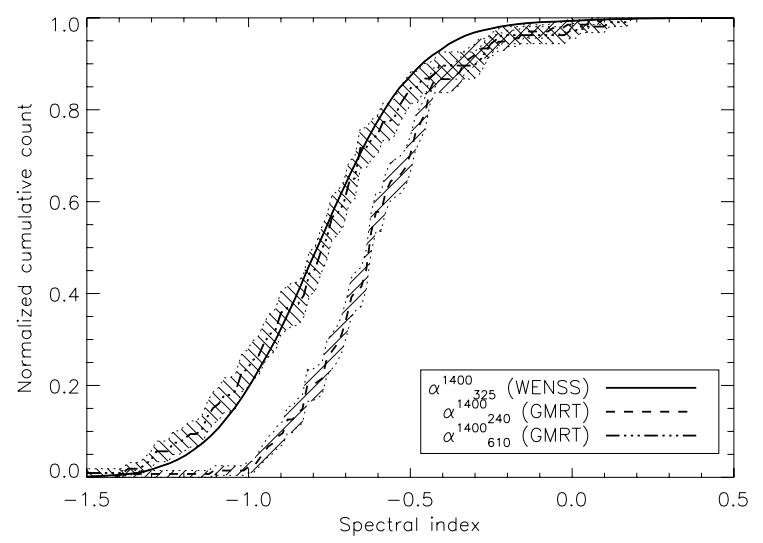

Fig. 5. The distribution of the spectral index $\alpha_{240}^{1400}$ and $\alpha_{610}^{1400}$ derived from flux comparison between our surveys flux density estimates at 240 and $610 \mathrm{MHz}$, and the NVSS at $1.4 \mathrm{GHz}$. The full line stands for the spectral index distribution $\alpha_{325}^{1400}$ of the radio sources in the WENSS radio survey (De Breuck et al. 2000). To match the $5 \sigma$ flux density limit of WENSS, we have applied a flux density cut $S_{240}^{\text {int }}>63$ mJy and $S_{610}^{\text {int }}>30 \mathrm{mJy}$ at $240 \mathrm{MHz}$ and $610 \mathrm{MHz}$ respectively.

Figure 6 shows the Euclidean normalized differential source counts at 240 and $610 \mathrm{MHz}$ as well as the WENSS differential source counts extrapolated from from $325 \mathrm{MHz}$ (Wieringa 1991). In principle, in order to derive reliable estimates of the differential counts over the whole flux density range, biases and incompleteness correction have to be taken into account: (i) the incompleteness introduced by the variation of the complete area with flux density (Fig. 3); (ii) the incompleteness due to the efficiency of the source finding algorithm SAD that decreases with the signal-to-noise ratio; and (iii) the bias introduced by SAD flux density overestimation at low flux densities (see Seymour et al. 2005, for a detailed discussion on these biases). All these biases occur at low flux densities. We do not correct for any of these but plot the $95 \%$ completeness level estimated from the noise maps. Above this flux density, the differential counts are reliable. At $240 \mathrm{MHz}$, the differential counts agree with the WENSS differential counts until a flux density level of $\sim 100 \mathrm{mJy}$. At lower fluxes, the factor of $\sim 2$ difference with the expected values could be due to cosmic variance: at $325 \mathrm{MHz}$ in the same field the differential source count shows the same offset with the Wieringa (1991) differential source count at $\gtrsim 20 \mathrm{mJy}$ (Tasse et al. 2006). At $610 \mathrm{MHz}$, the Euclidean normalized differential source counts follow the Wieringa (1991) extrapolated differential source counts, consistently with a mean spectral index value of $\alpha \sim-1$.

\section{Radio spectra analysis}

\subsection{The multi frequency radio sample}

For each individual source, when comparing the flux density estimates obtained at the different frequencies, several issues have to be considered.

First, the sky plane has been imaged using different instruments, at different frequencies. In each frequency band, the uv plane coverage can be significantly different from one observation to another. This can lead to a variation in the flux estimate, shorter baselines being sensitive to larger angular scale radio emission. After the RFI flagging step, the shortest baselines in the uv plane are $\sim 0.3 \mathrm{k} \lambda$ at 240 and $610 \mathrm{MHz}, \sim 0.5 \mathrm{k} \lambda$ at $325 \mathrm{MHz}, 0.2 \mathrm{k} \lambda$ at $74 \mathrm{MHz}$, and $\sim 0.05 \mathrm{k} \lambda$ at $1400 \mathrm{MHz}$, corresponding to angular scales of $\sim 11.5^{\prime}, 6.9^{\prime}, 20^{\prime}$ and $\sim 1.2^{\circ}$ respectively. The largest extended radio emission detected in the XMM-LSS field is the $\sim 1^{\prime}$ radio halo candidate described in Tasse et al. (2006) and is smaller than the maximum angular scale detectable at $325 \mathrm{MHz}$. This effect is therefore assumed to have a negligible impact on the integrated flux density estimates.

Secondly, through the various frequency bands, the size of the synthesized beam varies from $6.5^{\prime \prime}$ at $610 \mathrm{MHz}$ to $45^{\prime \prime}$ at $1.4 \mathrm{GHz}$. As described in Sect. 4.2, since the Gaussian fitting method may miss a significant part of the extended flux in some cases, we also extract the integrated flux density based on pixel values within a $60^{\prime \prime}$ diameter aperture (see Sect. 4.2). This second estimation corrects for that effect.

Finally, the overall flux density calibration is reliable on the level of $\sim 5 \%$ for the VLA and $\sim 10 \%$ for the GMRT. These systematics have been taken into account within the error bar calculation. We discuss the effect of these systematics on the radio spectra fits in Sect. 6.4.

In order to study the shape of the low frequency radio spectra, we build a 5-frequency band catalog on the basis of the source lists at 74, 240, 325, 610, and $1400 \mathrm{MHz}$ (Tasse et al. 2006; Condon et al. 1998), by associating their Gaussian fitted components that are closer than $60^{\prime \prime}$. Considering the sum of all the number density of these various radio surveys, this radius makes the probability of two components to be associated by mistake to be less than $2 \%$.

\subsection{Comparison with VLA data}

For testing the $610 \mathrm{MHz}$ source list flux densities, we select the ones being detected at 325 and $1400 \mathrm{MHz}$. In order to be not affected by the angular extend of the radio sources in any way, we select only the sources being unresolved in all frequency bands. We define the spectral curvature as $\alpha_{325}^{610}-\alpha_{610}^{1400}$. The spectral curvature expresses whether the synchrotron slope shows any difference on the two sides of the $610 \mathrm{MHz}$ point. In Fig. 7 we plot the spectral curvature as a function of the flux density at $610 \mathrm{MHz}$. The mean spectral curvature value is $\left\langle\alpha_{325}^{610}-\alpha_{610}^{1400}\right\rangle=-0.15$, becomes $\sim 0$ if we correct the overall flux density scale on the level of $6 \%$, in agreement with the $\sim 10 \%$ error on the flux scale used to calculate the error bars. At low fluxes though, we see the mean spectral curvature to reach negative values of $\sim-0.8$, which is due to a selection effect: we have selected the pointlike $610 \mathrm{MHz}$ sources being detected at both 325 and 1400 . Therefore at a given $610 \mathrm{MHz}$ flux density, and given the 325 and $1400 \mathrm{MHz}$ flux density limit (Fig. 3), a range of spectral curvature values is not observable. If we compute the observable spectral curvature range as a function of the $610 \mathrm{MHz}$ flux density, we clearly see how biased the spectral curvature measurement is for faint $610 \mathrm{MHz}$ sources.

We carry out the same analysis at $240 \mathrm{MHz}$ (Fig. 7, left panel). The mean spectral curvature value is $\left\langle\alpha_{240}^{325}-\alpha_{240}^{1400}\right\rangle=$ -0.11 corresponding to the $240 \mathrm{MHz}$ flux density being overestimated. Nevertheless, a correction of the overall flux density scale on the level of $\sim 4 \%$ brings the averaged spectral curvature value to $\sim 0$, in agreement with the $10 \%$ error on the gain calibration. The agreement between flux density estimates at 240 , 325 , and $1400 \mathrm{MHz}$ seems to hold until the $5 \sigma$ level although we note the presence of sources showing very low spectral curvature values. The bias introduced by the different flux density limits at 325 and $1400 \mathrm{MHz}$ do not seem to have much influence. That is because assuming a spectral index of -0.8 the $240 \mathrm{MHz}$ flux density limit is higher than the ones at 325 and $1400 \mathrm{MHz}$ by a factor af $\sim 2$ (see Fig. 3). 

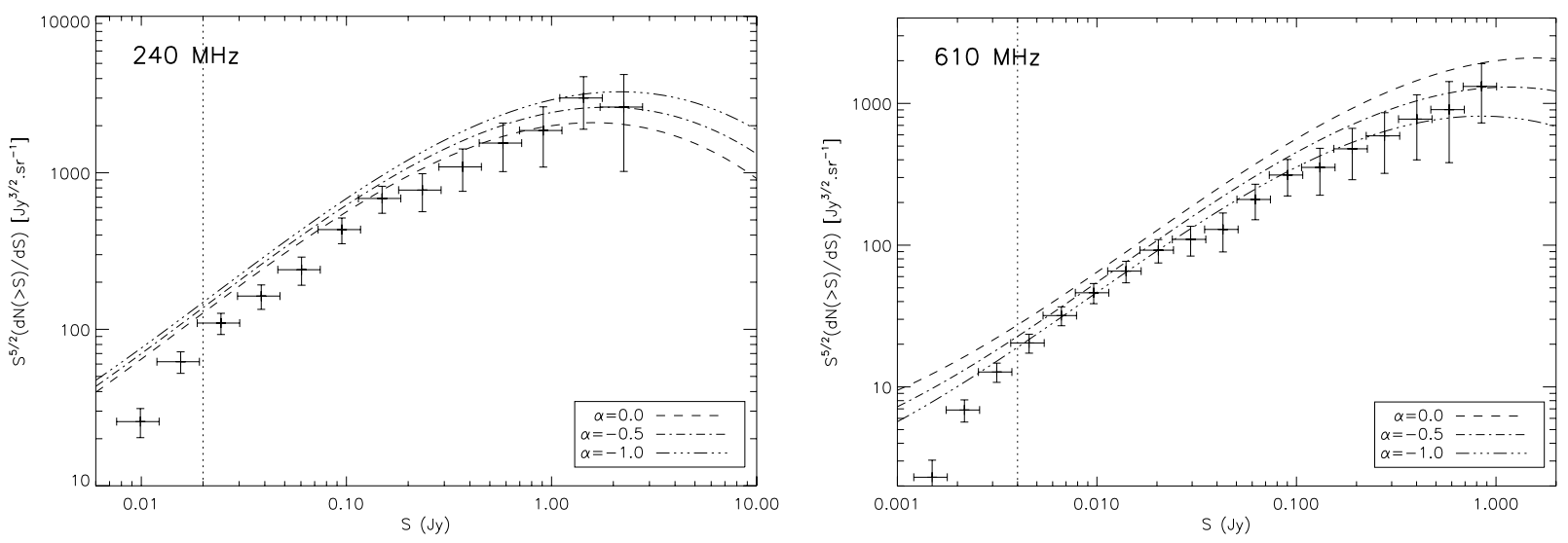

Fig. 6. Both panel show the Euclidean normalized differential source count at $240 \mathrm{MHz}$ (left panel), and $610 \mathrm{MHz}$ (right panel). The curves shows the extrapolated Wieringa (1991) $325 \mathrm{MHz}$ differential source count, assuming different spectral index values. The vertical dotted line show the $95 \%$ completeness level.
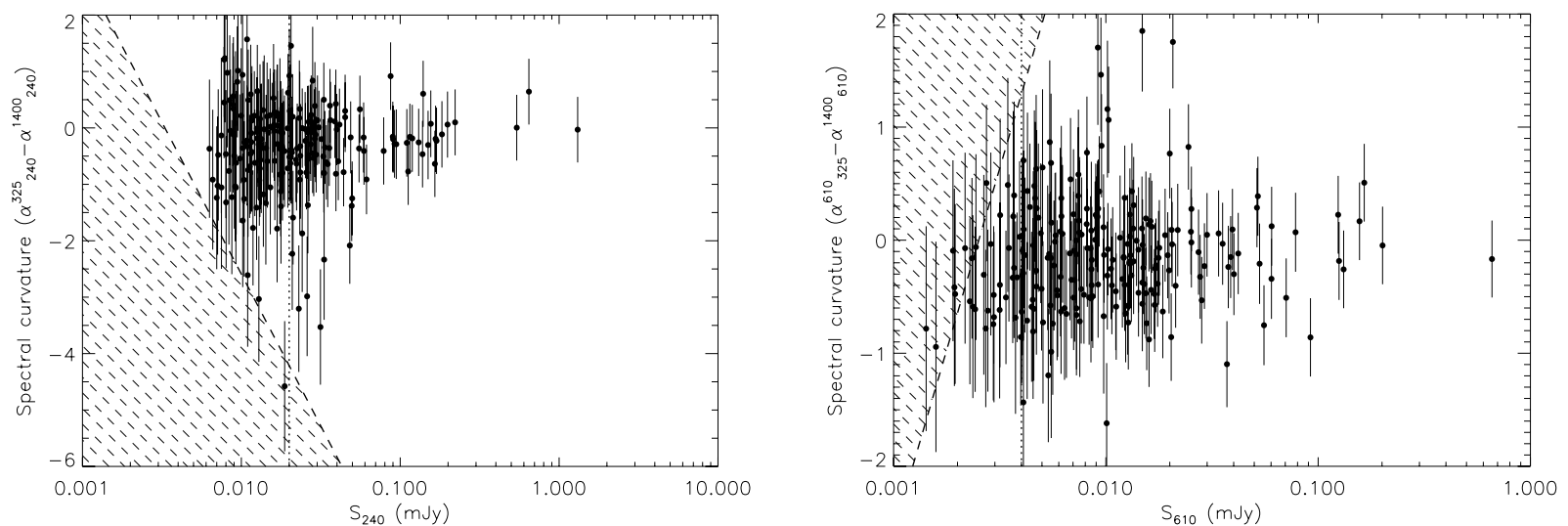

Fig. 7. These plots show the consistency of the flux density measurements of the VLA and the GMRT instruments. Left panel: for the sources detected at 240,325, and $1400 \mathrm{MHz}$, the spectral curvature $\alpha_{240}^{325}-\alpha_{240}^{1400}$ is plotted as a function of the flux density at $240 \mathrm{MHz}$. The overall shift is explainable in term of flux density calibration being accurate on the level of $\sim 10 \%$ on true spectral curvature. The right panel similarly shows the spectral curvature $\alpha_{325}^{610}-\alpha_{610}^{140}$ as a function of the $610 \mathrm{MHz}$ flux density. Because each survey is flux density limited, a range of spectral curvature is not reachable at a given 240 or $610 \mathrm{MHz}$ flux density level (dashed area). This explains the apparent decreasing of the spectral curvature at low $610 \mathrm{MHz}$ flux densities. Some points stand with the dashed area because the flux density limit is not homogeneous in the radio maps, both at 325 and $1400 \mathrm{MHz}$. Vertical dotted lines stand for the $95 \%$ completeness limit.

\subsection{Spectral fits}

Theoretically, the classical power law spectrum of synchrotron emission is understood as a zero aged relativistic electron population, having a power-law energy distribution, and being optically thin to its own radiation (Kardashev 1962). Deviation from the power law occurs with (1) a low frequency turnover due to synchrotron self absorption at low frequency and (2) a steepening at high frequencies due to particle energy losses. When the major fraction of the flux density is emitted by one, single electron population the radio spectra are well approximated by a simple synchrotron radiation model, including self absorption and spectral ageing features. The presence of different synchrotron emitting electron population will make the integrated radio spectra significantly deviate from the simple synchrotron spectrum. For example FRI radio sources (Fanaroff \& Riley 1974) show a compact flat spectrum core and steep spectrum extended lobes.

For a crude modeling of the synchrotron emission, we approximate the integrated radio spectra to be a emitted by a single electron population, following the continuous injection model described above. It is well known that under these assumptions the synchrotron spectrum $S(v)$ has a standard shape (Kardashev 1962), modified by low frequency self-absorption (Pacholczyk 1970) as follows:

$$
\begin{aligned}
& S(v)=S_{0}\left(v / v_{1}\right)^{5 / 2-\alpha}\left(1-\mathrm{e}^{-\left(v / v_{1}\right)^{\alpha-5 / 2)}}\right) S_{\mathrm{aged}} \\
& S_{\text {aged }}= \begin{cases}v^{\alpha} & : v<v_{\mathrm{c}} \\
v^{\alpha-1 / 2} & : v>v_{\mathrm{c}}\end{cases}
\end{aligned}
$$

where $S_{0}$ is a normalization factor, $v_{1}$ is the self absorption frequency break, $\alpha$ is the zero aged spectral index in the transparent frequency range and $v_{\mathrm{c}}$ is the break frequency above which the power-law breaks to be spectrally aged. Our free parameter space for the spectral fit is composed of $\left\{S_{0}, \alpha, v_{1}, v_{\mathrm{c}}\right\}$. The initial condition of the fitting routine are set on a $15 \times 21 \times$ $21 \times 21$ parameter grid. The $v_{1}$ and $v_{\mathrm{c}}$ initial condition ranges are $\sim 50-250 \mathrm{MHz}$ and $\sim 300-2000 \mathrm{MHz}$ respectively whereas the initial condition ranges for $S_{0}$ and $\alpha$ depend on individual flux density levels and error bars.

\subsection{Subsample definition}

Because we want to build samples of sources showing clear signatures of spectral ageing and/or self-absorption, we select in the 

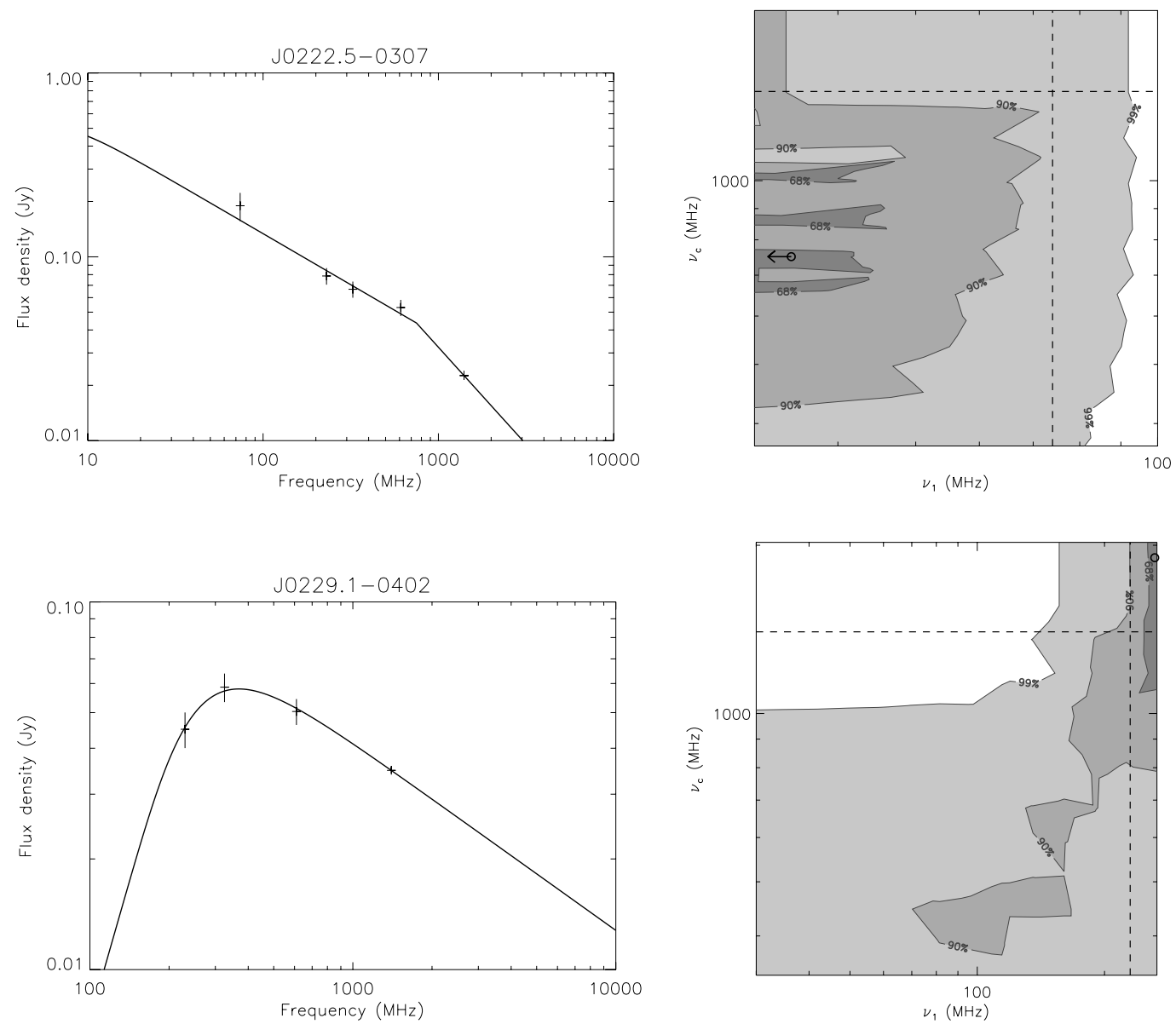

Fig. 8. Examples of two radio sources, showing spectral ageing (top) and self absorption signatures (bottom). Left panels show the flux density measurement at 74, 240,325, 610 and $1400 \mathrm{MHz}$ together with their associated error bars. The full line shows the best fit spectrum described by Eqs. (4) and (5). The right panels show the dependence of the $\chi^{2}$ on the parameters $v_{1}$ and $v_{\mathrm{c}}$, that are used for the radio spectral type classification. The parameters corresponding to the best fit is marked with a thick open circle. The different contour lines show the $\chi_{\min }^{2}+1, \chi_{\min }^{2}+2.71$ and $\chi_{\min }^{2}+6.63$ levels corresponding to the $68 \%, 90 \%$ and $99 \%$ confidence intervals respectively (Avni 1976). The vertical and horizontal dashed lines correspond to the lowest and highest available frequency respectively. In the final fitted parameters table, we consider the best fit parameter together with the $68 \%$ confidence interval covering also the degenerated solution. In these examples, based on the $68 \%$ confidence interval for $v_{1}$ and $v_{\mathrm{c}}$, we can see the model fits the flux density points only if spectral ageing and self-absorption are present in the first and second case respectively.

multi radio frequency catalog described in Sect. 6.1 the objects having been detected at least at 240, 325, 610, and $1400 \mathrm{MHz}$, which makes the total number of fitted sources to be $318(38 \%$ of the sources detected at $325 \mathrm{MHz}$ ). When the object is not detected at $74 \mathrm{MHz}$, we have used the flux limit at $74 \mathrm{MHz}$ to constrain the fitting parameters.

In order to reject the objects that could not be well fitted we choose a reduced ${ }^{6} \chi^{2}$ cut above which the model described with Eq. (5) is assumed to be irrelevant. Figure 9 shows that if we select the spectral fits with an associated $\chi_{\text {reduced }}^{2}<4.3$ then the observed $\chi_{\text {reduced }}^{2}$ distribution follows the theoretical distribution ${ }^{7}$, meaning we can consider the model to be relevant for these objects. We visually inspected the radio images of the objects having an associated $\chi_{\text {reduced }}^{2}>4.3(\sim 25 \%$ of the selected sample).

${ }^{6}$ To calculate the reduced $\chi^{2}$ we have used the formula $\chi_{\text {reduced }}^{2}=$ $\chi_{\min }^{2} /(n-p)$ where $n$ if the number of data points and $p$ is the number of parameter in the model. The value of $p$ depends on whether the parameters $v_{\mathrm{c}}$ and $v_{1}$ of Eq. (5) have to be used to fit the radio spectra. For example if we use a single power law then $p=2$.

7 The reduced $\chi^{2}$ distribution theoretically follows a normal distribution with one degree of freedom.
They are found to be mainly resolved, having a few to many radio emitting components. For these objects, the assumptions of a single synchrotron emitting electron population (Sect. 6.3) is wrong, and the simple model we have used is most probably irrelevant. In order to be conservative, we list the fitted parameters for all objects in Table B.3 (including the $\chi_{\text {reduced }}^{2}>4.3$ objects).

The minimum $\chi^{2}$ is recorded in the 4-parameter space, and the errors on the fitted parameters are derived from the $68 \%$ confidence interval calculated at $\chi_{\min }^{2}+1$ (Avni 1976). In the case of degenerated solutions, instead of considering the list of all the $\chi^{2}$ minima with their respective associated error bars, only the best fit parameters are considered, and the error bar associated with them is the interval including all the multiple $\chi^{2}$ minima (See Fig. 8). This method allows us to define a robust sample of sources having detected self absorption of spectral ageing feature. A source is considered to be showing self absorption if and only if the $68 \%$ confidence interval of $v_{1}$ falls within the frequency range $\left[v_{\text {low }}, 1.4 \mathrm{GHz}\right]^{8}$ probed by our survey, meaning

${ }^{8}$ Because we have the objects detected in at least 4 bands, the lower available frequency $v_{\text {low }}$ value depends on whether the object is detected at $74 \mathrm{MHz}$. In all cases, if this is not the case then $v_{\text {low }}=240 \mathrm{MHz}$. 


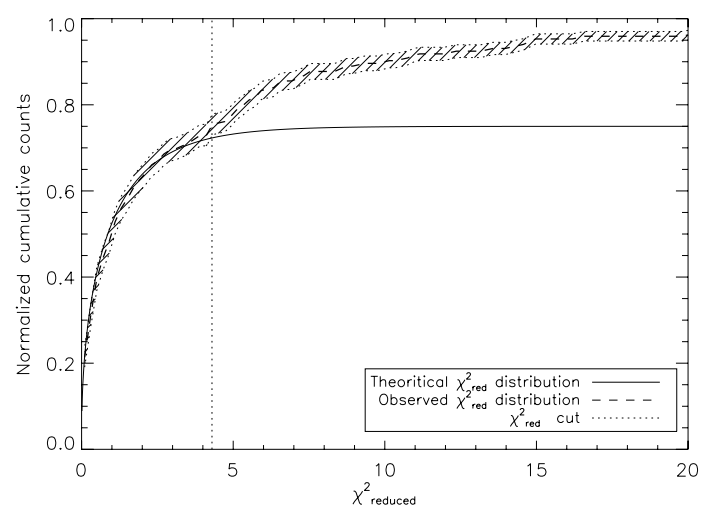

Fig. 9. The cumulative distribution of the reduced $\chi^{2}$. Choosing a $\chi_{\text {red }}^{2}$ cut of 4.3 , we see that $\sim 75 \%$ of the population behaves like the ajusted theoretical $\chi_{\text {red }}^{2}$ distribution (black line).

good solutions cannot be found without observed self absorption. We proceed the same way for the $v_{\mathrm{c}}$ free parameter. This allows us to define robust subsamples of radio spectra showing self absorption, or spectral ageing or both.

The best fit parameters with their associated $68 \%$ confidence interval as well as their classification appear in Table B.3.

On the subsample of fitted spectra, $\sim 68 \%$ are found to show no significant signs of spectral ageing or self absorption, $\sim 6 \%$ show self absorption only, $\sim 26 \%$ show spectral ageing only, and none show both. It has been suggested in the past that compact steep spectrum (CSS) and gigahertz peaked spectrum (GPS) may correspond to the early stages of evolution of classical FRI and FRII radio sources, consistent with the observed subarcsecond angular size of CSS and GPS radio sources (Fanti et al. 1995; O'Dea \& Baum 1997; Snellen et al. 2000). Conversely, the radio sources showing spectral ageing in their radio spectra are the ones in which no high energy electron is injected into the radio emitting lobes. These radio sources should correspond to the latest evolutionary stage of radio sources. In order to test the consistency of the three radio spectral classes ${ }^{9}$, we compare their respective angular size distribution. As the resolution at 325 and $610 \mathrm{MHz}$ is higher, we estimate the angular size of the objects in the sample based on the Gaussian fitting components positions and their elongation deconvolved from the beam size at these two frequencies. Figure 10 shows the cumulative distribution of the angular sizes for the three subsample. $\sim 60 \%$ of the steep spectrum sources are unresolved, compared to $\sim 40 \%$ and $\sim 80 \%$ for the sources showing signatures of spectral ageing and self-absorption respectively. Consistently, the distribution of spectrally aged sources is clearly biased towards sources having larger angular sizes than the single power law sources by a factor of $\sim 1.5-2$. In the subsample of sources showing signs of self absorption, we note the presence of three sources having large angular diameters $\gtrsim 10^{\prime \prime}$ (J0217.0-0308, J0218.3-0403, J0219.7-0448) ${ }^{10}$.

In principle we should be allowed to compare a parameter distribution only for a homogeneously selected sample.

\footnotetext{
9 Steep spectrum, Self absorbed and Spectrally aged.

10 Detailed inspection of the radio images at 240,325 and $610 \mathrm{MHz}$, show that the extended source J0217.0-0308 has its $240 \mathrm{MHz}$ position in between two facets, likely making the flux to be badly estimated and the source to be classified as self absorbed. J0218.3-0403 and J0219.7-0448 have an FRI-like morphology, suggesting the flux density of the central component might dominates the overall flux density emission. Self absorption might therefore happen within the central core of these sources although they are measured to be extended.
}

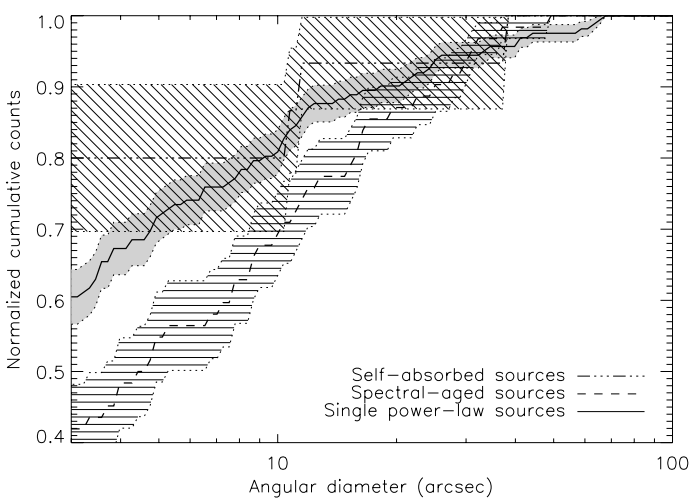

Fig. 10. The angular diameter of the radio sources classified as spectrally aged or self absorbed. Spectrally aged sources appear larger on average than single power law sources and self-absorbed sources.

Nevertheless, we have built subsamples of radio sources based on their radio spectral shape, selecting the objects that are detected at $240,325,610$, and $1400 \mathrm{MHz}$ at least, the various surveys having different flux density limits (see Fig. 3). For example let us consider a source at a given redshift and $325 \mathrm{MHz}$ luminosity: in the observer frame, a self absorbed source is more likely not to appear in the subsamples because its $240 \mathrm{MHz}$ flux density may be below the flux density limit of the associated survey. A similar effect acts on the spectrally aged subsample. These preliminary results on the angular size distribution in the various radio source classes have to be taken with caution because selection effect may play an important role.

\section{Conclusion and future work}

We have imaged the low frequency $325 \mathrm{MHz}$ counterpart (Tasse et al. 2006) of the XMM-LSS field at 240 and $610 \mathrm{MHz}$ with the GMRT, leading to the detection of 467 and 667 radio sources respectively. The GMRT survey covers 18.0 degree $^{2}$ at $240 \mathrm{MHz}$ and 12.7 degree $^{2}$ at $610 \mathrm{MHz}$, with average flux density limits of 12.5 and $1.5 \mathrm{mJy} /$ Beam $(5 \sigma)$ respectively. We have corrected for the various systematic errors introduced by the instrument and the data reduction procedure on both astrometry and flux density scale. Comparison between the catalogs at 240 and $610 \mathrm{MHz}$ with other datasets at 325 , and $1400 \mathrm{MHz}$ show good agreement, although the spectral index distribution suggests a spectral index flattening below $240 \mathrm{MHz}$.

We have fitted a simple continuous injection synchrotron emission model to the flux density measured at 74 and $325 \mathrm{MHz}$ by Tasse et al. (2006), 240 and $610 \mathrm{MHz}$ presented in this paper, and 1.4 GHz (NVSS, Condon et al. 1998). On the basis of fitted parameters and error bars estimates, we define a sample of radio sources showing signatures of self absorption or spectral ageing feature in their radio spectra. Consistent with other studies suggesting a link between radio spectra shape and radio source evolution, we found that spectrally aged sources have larger angular sizes, whereas sources showing self-absorption are smaller.

In the near future, on the basis of the available X-ray and optical data we will study the effect of environment on the fundamental properties of the various classes of radio sources.

Acknowledgements. We thank the staff of the GMRT that made these observations possible. GMRT is run by the National Center for Radio Astrophysics of the Tata Institute of Fundamental Research. We thank Ishwara Chandra, Walter Jaffe, Dave Green, Niruj Mohan Ramanujam, Amitesch Omar, Ignas Snellen for useful discussion on the data reduction and analysis. 


\section{Appendix A: Radio images}
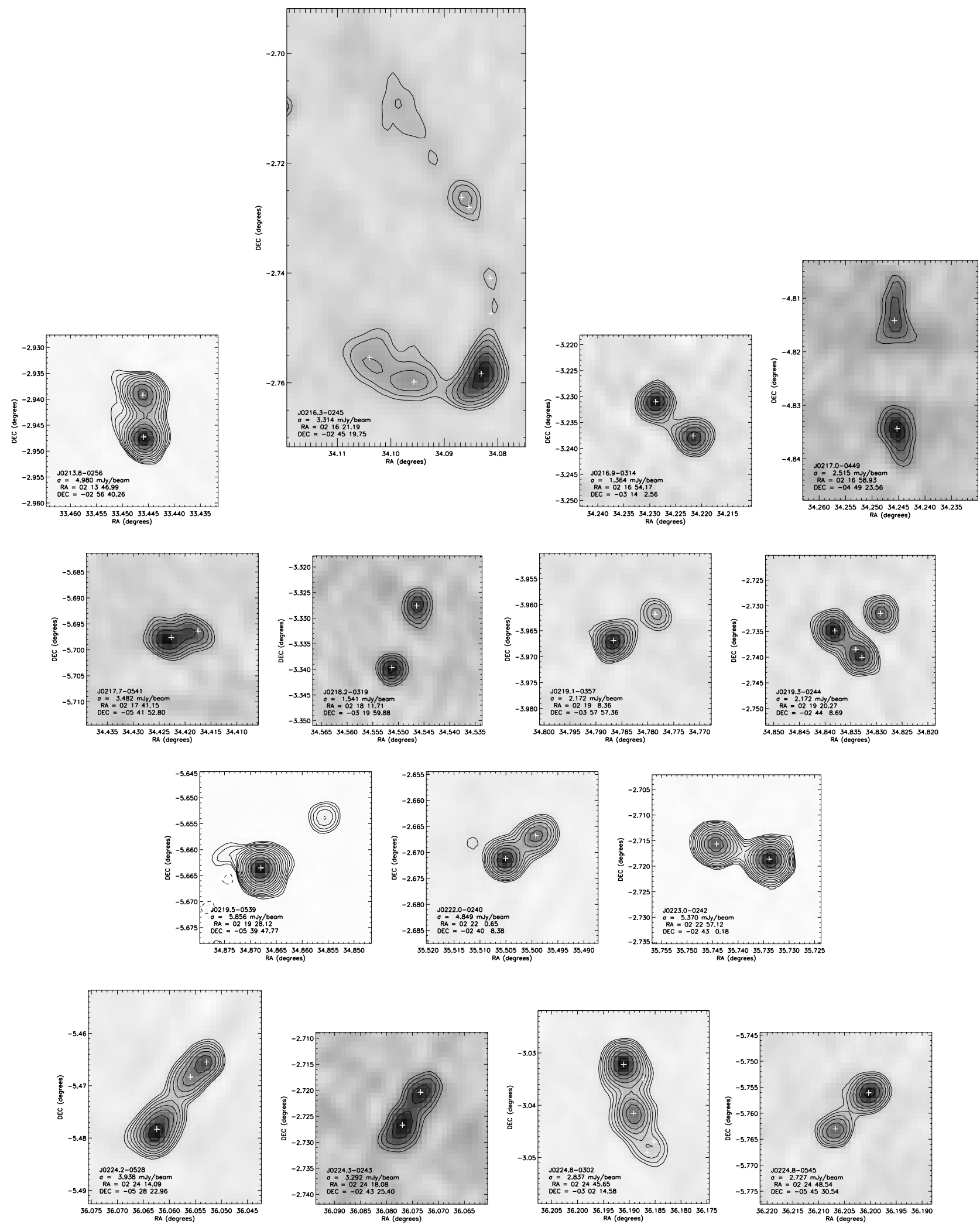

Fig. A.1. At $240 \mathrm{MHz}$, the 20 largest sources. Sorting order is increasing right assenssion. Contours corresponds to levels of $3 \sigma \times$ $(-1.4,-1,1,1.4,2,2.8,4,5.6,8,11, \ldots)$ and greyscale is scaled from $-3 \sigma$ to the maximum value in the image. On the bottom-left corner of each image appears the name of the corresponding source in the source list, its flux weighted coordinate as well as the local noise level. The crosses are marking the Gaussian fitting components. 

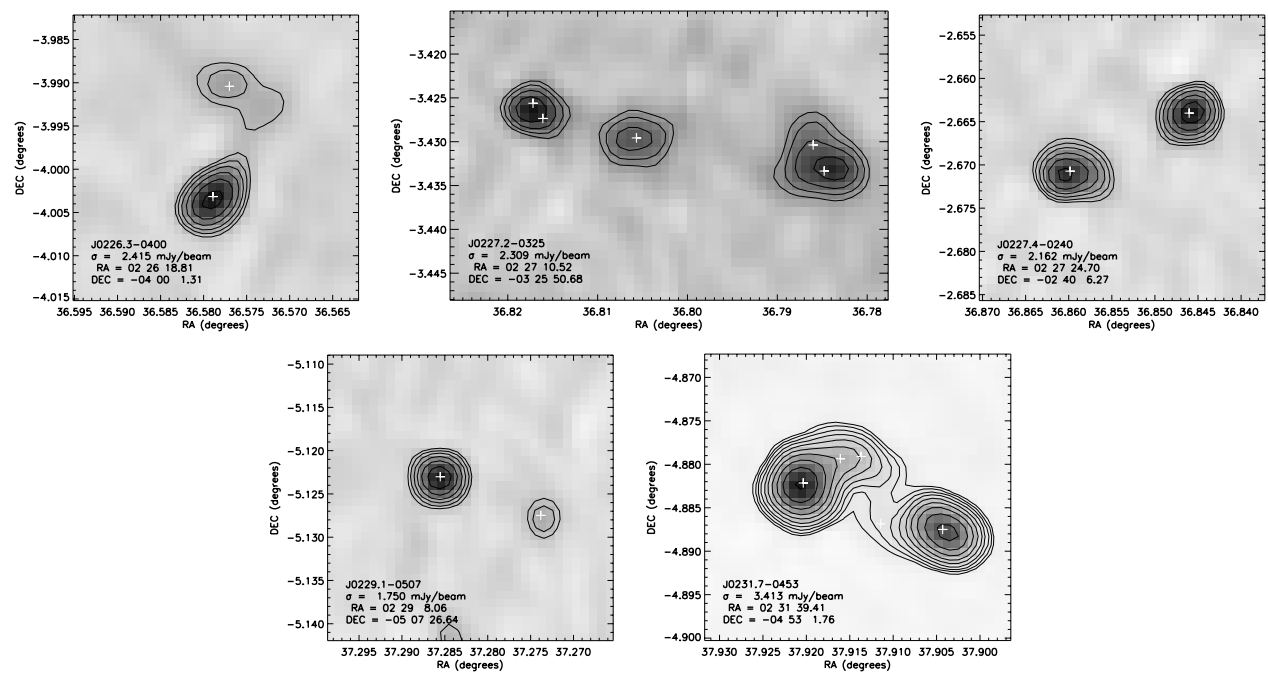

Fig. A.1. continued.
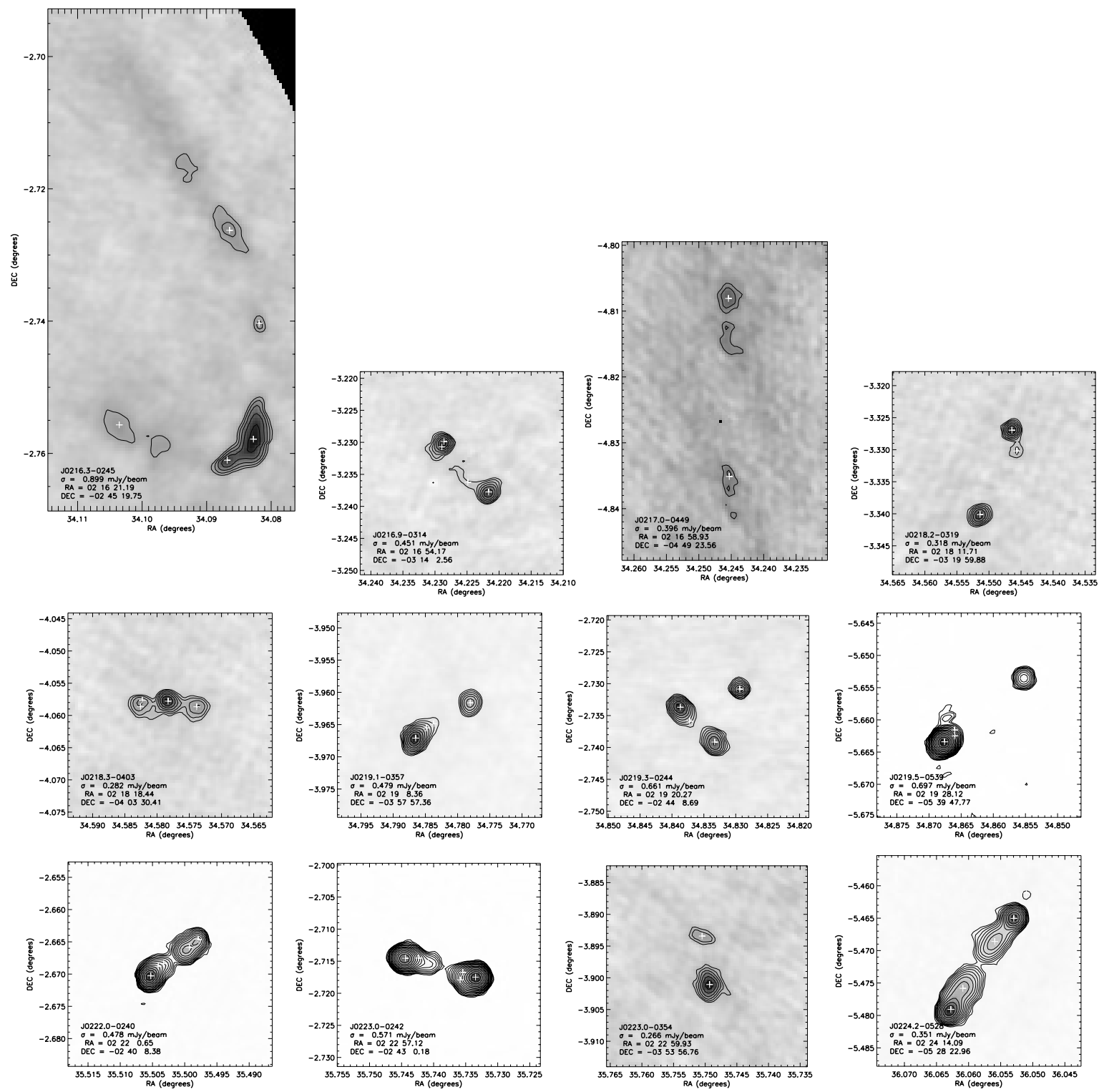

Fig. A.2. At $610 \mathrm{MHz}$, the 20 largest sources. Sorting order is increasing right assenssion. Contours corresponds to levels of $3 \sigma \times$ $(-1.4,-1,1,1.4,2,2.8,4,5.6,8,11, \ldots)$ and greyscale is scaled from $-3 \sigma$ to the maximum value in the image. On the bottom-left corner of each image appears the name of the corresponding source in the source list, its flux weighted coordinate as well as the local noise level. The crosses are marking the Gaussian fitting components. 

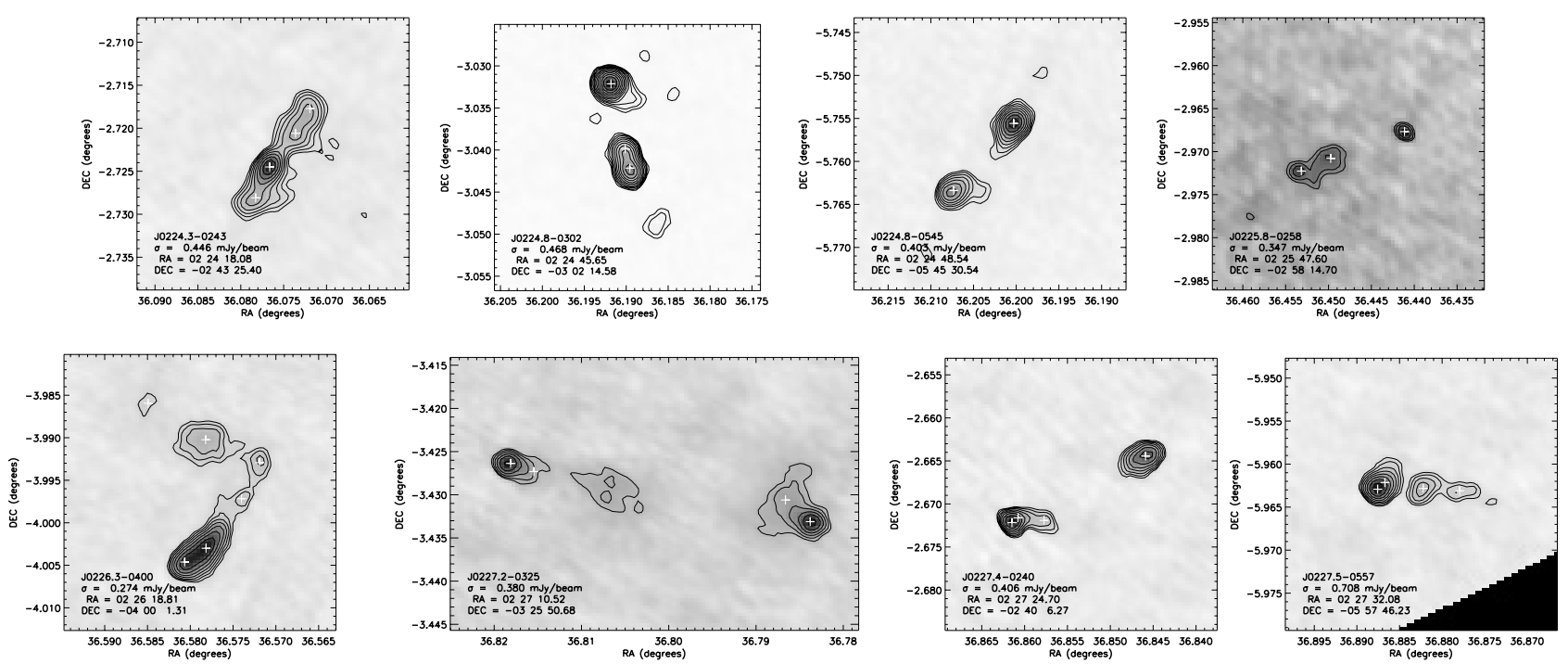

Fig. A.2. continued.

\section{References}

Avni, Y. 1976, ApJ, 210, 642

Becker, R. H., White, R. L., \& Helfand, D. J. 1995, ApJ, 450, 559

Chandra, P., Ray, A., \& Bhatnagar, S. 2004, ApJ, 612, 974

Cohen, A. S., Röttgering, H. J. A., Kassim, N. E., et al. 2003, ApJ, 591, 640

Condon, J. J. 1997, PASP, 109, 166

Condon, J. J., Cotton, W. D., Greisen, E. W., et al. 1998, AJ, 115, 1693

De Breuck, C., van Breugel, W., Röttgering, H. J. A., \& Miley, G. 2000, A\&AS, 143,303

Fanaroff, B. L., \& Riley, J. M. 1974, MNRAS, 167, 31P

Fanti, C., Fanti, R., Dallacasa, D., et al. 1995, A\&A, 302, 317

Haslam, C. G. T., Salter, C. J., Stoffel, H., \& Wilson, W. E. 1982, A\&AS, 47, 1

Kardashev, N. S. 1962, Sov. Astron., 6, 317

Lonsdale, C. J., Smith, H. E., Rowan-Robinson, M., et al. 2003, PASP, 115, 897

Mohan, R., Dwarakanath, K. S., Srinivasan, G., \& Chengalur, J. N. 2001, J.A\&A, 22,35
O’Dea, C. P. 1998, PASP, 110, 493

O'Dea, C. P., \& Baum, S. A. 1997, AJ, 113, 148

Pacholczyk, A. G. 1970, Radio astrophysics. Nonthermal processes in galactic and extragalactic sources (Series of Books in Astronomy and Astrophysics, San Francisco: Freeman)

Perley, R. A. 1999, in Synthesis Imaging in Radio Astronomy II, ASP Conf. Ser., 180,383

Pierre, M., Valtchanov, I., Altieri, B., et al. 2004, J. Cosm. Astro-Particle Phys., 9, 11

Rengelink, R. B., Tang, Y., de Bruyn, A. G., et al. 1997, A\&AS, 124, 259

Seymour, N., McHardy, I., Gunn, K., \& Moss, D. 2005, in The Dusty and

Molecular Universe: A Prelude to Herschel and ALMA, ed. A. Wilson, 323

Snellen, I. A. G., Schilizzi, R. T., Miley, G. K., et al. 2000, MNRAS, 319, 445

Snellen, I. A. G., Lehnert, M. D., Bremer, M. N., \& Schilizzi, R. T. 2003, MNRAS, 342, 889

Tasse, C., Cohen, A. S., Röttgering, H. J. A., et al. 2006, A\&A, 456, 791

Wieringa, M. H. 1991, Ph.D. Thesis 